\title{
Heart failure with preserved ejection fraction
}

\section{A growing global epidemic}

Pyi Naing, Douglas Forrester,

Nadarajah Kangaharan,

Aruna Muthumala, Su Mon Myint,

David Playford

\section{Background}

Heart failure with preserved ejection fraction ( $\mathrm{HFpEF}$ ) is an emerging global health problem of which there is limited awareness. HFpEF has a prognosis similar to that of heart failure with reduced ejection fraction (HFrEF) and accounts for approximately half of all patients with heart failure.

\section{Objective}

The aim of this article is to review $\mathrm{HFpEF}$ and its consequences and management, including examples of patients with HFpEF.

\section{Discussion}

Patients with HFpEF may present with dyspnoea, fluid retention, lethargy and dizziness, making it difficult to differentiate clinically from HFrEF. The risk factors include increasing age, obesity, hypertension, diabetes, chronic kidney disease and obstructive sleep apnoea. The diagnosis requires good clinical acumen combined with echocardiography and elevated plasma B-type natriuretic peptide concentration. Management of HFpEF, especially in later stages, is difficult as there is no evidence-based therapy to date. Prevention is the best strategy. Early recognition and diagnosis are also very important to tackle this global epidemic.
HEART FAILURE WITH PRESERVED EJECTION FRACTION (HFpEF) is a clinical syndrome in which patients have clinical features of heart failure in the presence of normal or near-normal left ventricular ejection fraction, usually defined as ejection fraction at $50 \%$ or above. ${ }^{1} \mathrm{HFpEF}$ is not a single condition but a result of many different pathologies, adding challenges to management. HFpEF is a rapidly growing problem due to an ageing population as well as increased incidence of risk factors such as hypertension, diabetes and obesity (Figure 1). It can potentially become a major burden to healthcare systems worldwide, which are already overstretched.
Unlike impaired ejection fraction, which reliably defines heart failure with reduced ejection fraction (HFrEF), the diagnosis of $\mathrm{HFpEF}$ can be challenging as there is no single abnormal echocardiographic parameter that defines its presence. A diagnosis relies on thorough interpretation of the entire echocardiogram, including left ventricular mass, left atrial volume and assessment of diastolic function. ${ }^{2}$

\section{Pathophysiology and aetiology} The most common cause of HFpEF is left ventricular diastolic dysfunction secondary to a stiff, hypertrophied left ventricle causing elevated left

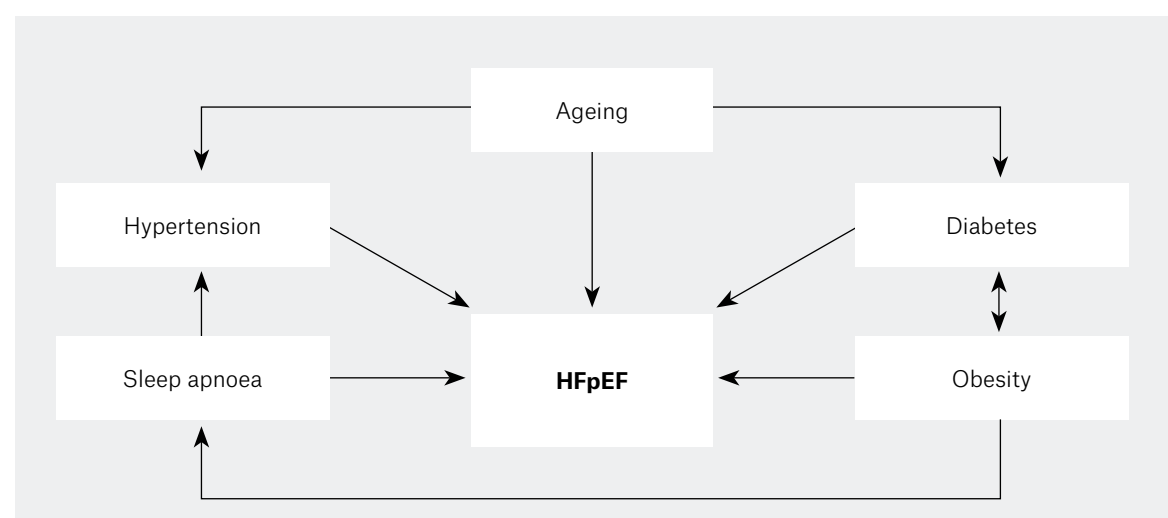

Figure 1. Heart failure with preserved ejection fraction and its risk factors (comorbid conditions), which are interlinked $H F p E F$, heart failure with preserved ejection fraction 
ventricular filling pressure (Figure 2). A classic example of this is left ventricular hypertrophy (LVH) caused by longstanding hypertension, in which chronic systemic pressure overload causes increased left ventricular muscle mass. This in turn causes myocardial ischaemia, which occurs due to the degree of hypertrophy outstripping the myocardial blood supply, resulting in fibrosis and stiffness of the left ventricle. In some patients with HFpEF, a smaller left ventricular cavity resulting from concentric hypertrophy can cause a decrease in stroke volume despite a deceptively normal ejection fraction. Severe aortic stenosis produces LVH in a similar fashion to longstanding severe systemic hypertension.

In contrast, patients with infiltrative cardiomyopathies such as cardiac
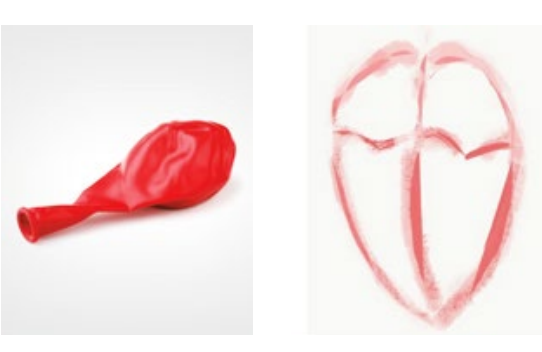

- Normal left ventricle behaves like a compliant rubber balloon that is easy to fill even by mouth

- There is also active left ventricular relaxation (dependent on adenosine triphosphate) in early diastole causing suction effect on the left atrial blood
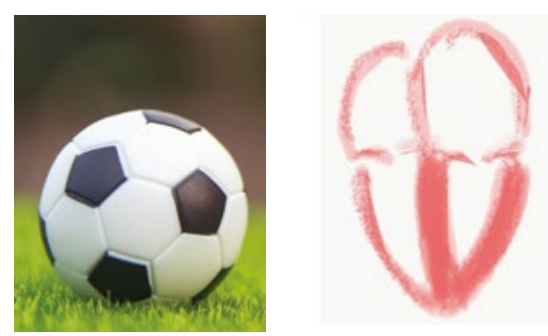

- Stiff left ventricle in patients with heart failure with preserved ejection fraction acts as a football that requires more pressure to fill (an air pump is needed)

- Loss of active diastolic relaxation leads to reliance on high left atrial pressure to push blood into the left ventricle, which in turn causes pulmonary venous hypertension and pulmonary congestion

- Reduced left ventricular end diastolic volume causes reduced stroke volume

Figure 2. Simplified explanation of heart failure with preserved ejection fraction using the balloon and football analogy

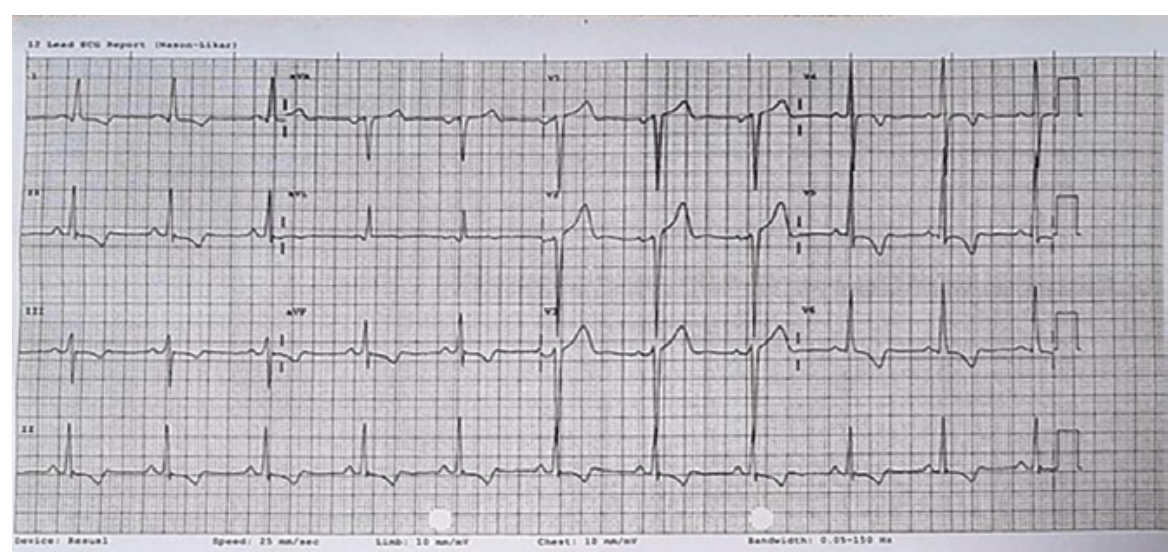

Figure 3. Electrocardiogram showing left ventricular hypertrophy strain pattern: Downsloping ST depression and T inversion in lateral leads as well as deep S wave in septal leads

amyloidosis, sarcoidosis and haemochromatosis may initially present with HFpEF, although the cause is clearly different from patients with hypertrophy caused by systemic hypertension. Right heart failure and heart failure due to valvular and pericardial diseases are also classified as HFpEF in some clinical trials and registries, but these conditions are heterogeneous and do not share similar pathophysiology nor outcomes. We will not consider these conditions further in this review.

\section{Prevalence and prognosis}

Prevalence of HFpEF in the community was reported in 2042 randomly selected individuals older than 45 years of age in Olmsted County, Minnesota, USA. ${ }^{3}$ The study found that approximately one in four individuals had some degree of diastolic dysfunction. Moderate and severe diastolic dysfunction (affecting 6.6\% and $0.7 \%$ of the studied population, respectively) were associated with a significant risk of mortality: approximately $23 \%$ mortality at five years. Those with mild diastolic dysfunction, representing $20.8 \%$ of the studied population, had $10 \%$ mortality at five years. It was also estimated that $50 \%$ of patients with HFpEF will develop pulmonary hypertension, a condition with a very poor prognosis with estimated medium survival of approximately four years from diagnosis. ${ }^{4,5}$ These data illustrate not only the magnitude of the problem, but also the imperative that primary care physicians are fully aware of how to make this important diagnosis.

Among the patients who were admitted to Mayo clinic hospitals with acute heart failure within the 15-year period, 53\% had reduced ejection fraction and $47 \%$ had preserved ejection fraction. ${ }^{6}$ At the medium-term follow-up of 10 years, survival rates were similar, with mortality rates of $29 \%$ and $32 \%$ at one year, and $65 \%$ and $68 \%$ at five years for $\mathrm{HFpEF}$ and HFrEF, respectively. Cohort studies have reported the chronic heart failure prevalence in the Australian general practice setting ranges from $3.6 \%$ to $4.3 \% ;^{7,8}$ however, it may be significantly underestimated, as HFpEF is notoriously underdiagnosed. ${ }^{9}$ 


\section{Clinical diagnosis and laboratory investigations}

Patients with HFpEF usually present with clinical features of heart failure such as dyspnoea, reduced exercise tolerance and pulmonary and systemic congestion. It is difficult to clinically differentiate HFpEF from HFrEF, although systemic hypertension, older age, female sex, obesity, atrial fibrillation and a lack of ischaemic heart disease history is more likely in HFpEF. ${ }^{10,11}$

The electrocardiogram (ECG) in patients with HFpEF may show evidence of $\mathrm{LVH}$, such as tall $\mathrm{R}$ waves in lateral leads and deep $S$ waves in septal leads, and changes of endocardial ischaemia ('strain'), as shown in Figure 3. In contrast, amyloid infiltration causes paradoxically low-voltage ECG despite an increased left ventricular mass in cardiac amyloidosis. Cardiac conduction problems in infiltrative cardiomyopathies may manifest as varying degree of heart blocks. Chest X-rays (CXR) of patients with HFpEF do not typically show cardiomegaly, but left atrial enlargement (splaying of carina on CXR) may be noticeable. Plasma B-type natriuretic peptide (BNP) levels are also raised in patients with $\mathrm{HFpEF}$ because of the stretch of myocardial muscle fibres, but BNP is nonspecific and cannot separate left and right heart causes, and is increased by renal failure and decreased in obesity. BNP levels of patients with HFpEF may be lower than those of patients with HFrEF because there is less end-diastolic wall stress in HFpEF. ${ }^{12,13}$ The BNP levels may also be normal in some patients with HFpEF, especially when patients are symptom-free. ${ }^{13}$

\section{Echocardiographic assessment of diastolic function}

Echocardiography is the most important investigation for the detection of diastolic dysfunction. ${ }^{2,14}$ Echocardiography is particularly useful to diagnose diastolic dysfunction as it provides anatomical as well as haemodynamic information. It is readily available and has no known side effects other than local irritation caused by pressure and minor local heating of tissues. Using two-dimensional echocardiography, LVH and left atrial dilatation can be reliably identified (Figure 4B). Echocardiography is also useful to identify valvular heart disease and left ventricular systolic dysfunction. Several Doppler echocardiographic parameters can be measured and calculated to determine the diastolic function. The pattern of transmitral blood flow from the left atrium to the left ventricle can be recorded by Doppler echocardiography (pulsedwave) and used to interpret diastolic function. The ratio between the early diastolic filling velocity ( $\mathrm{E}$ wave) and atrial systolic wave velocity (A wave) is a useful parameter to determine diastolic function. There are distinct patterns of
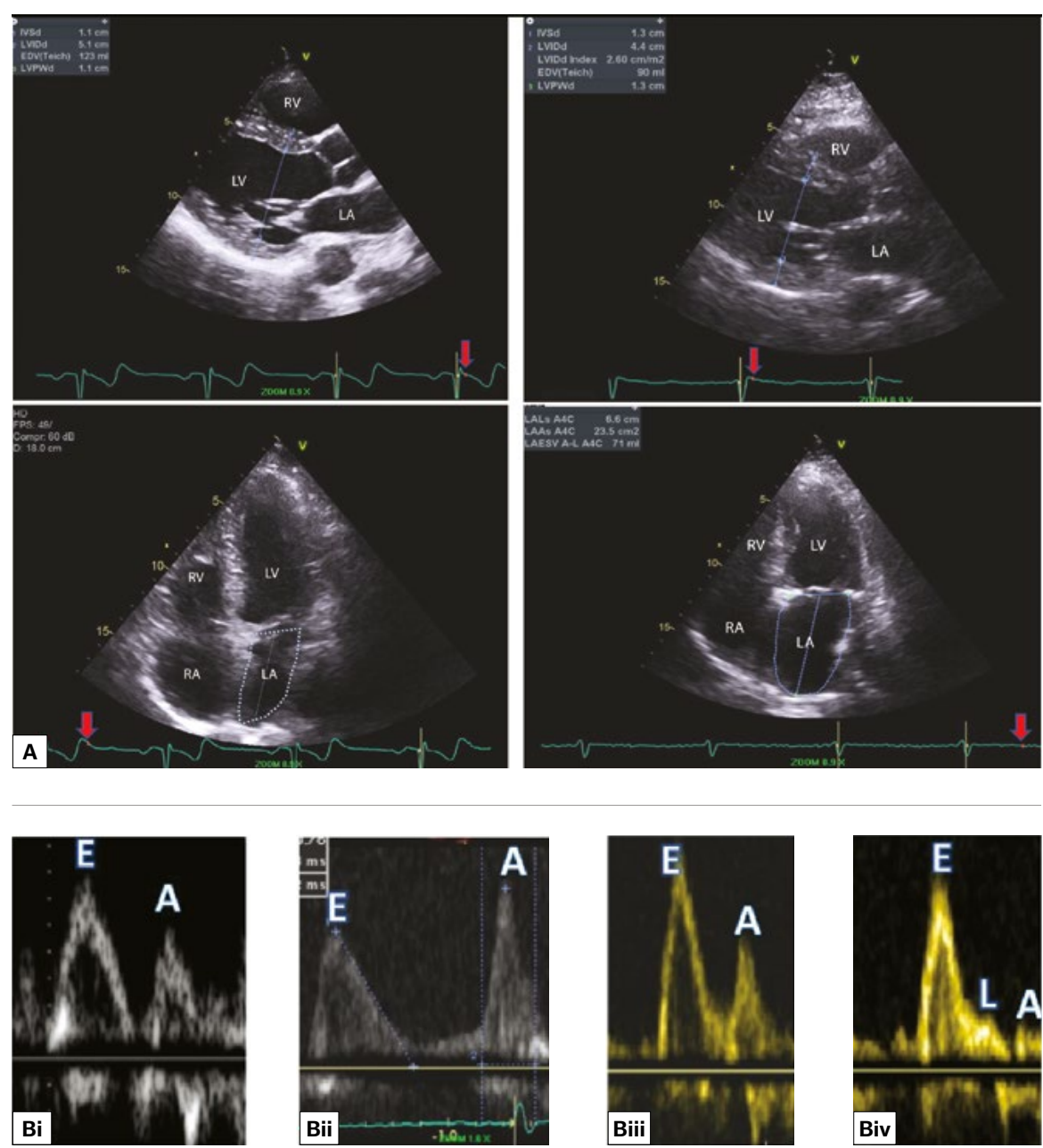

Figure 4 A. Side-by-side comparison of two-dimensional echocardiograms from a healthy person (left panel) and a patient with diastolic dysfunction (right panel); the upper images were captured in end diastole comparing normal versus thick left ventricular walls; the lower images were captured in end systole comparing the left ventricular and atrial sizes; B. Different patterns of early diastolic filling ( $E$ wave) and atrial systole (A wave) correspond with different degrees of diastolic function; Bi. Normal diastolic filling $(E>A)$; $\mathbf{B i i}$. Impaired relaxation $(E<A)$ : mild diastolic dysfunction; Biii. Pseudo normal pattern $(E>A)$ : moderate diastolic dysfunction; can be unmasked to impaired relaxation pattern with Valsalva manoeuvre; Biv. Restrictive filling pattern $(E / A>1.5)$ and $L$ wave: severe diastolic dysfunction.

$L V$, left ventricle; $L A$, left atrium; $R V$, right ventricle; $R A$, right atrium 
E and A waves depending on the degree of left ventricular diastolic dysfunction (Figure 4B).

Another useful Doppler measurement is the early diastolic mitral annular velocity (e') obtained with tissue Doppler (Figure 5Cii). The velocity of mitral annulus diastolic movement at both septal or lateral sides can be measured to determine the diastolic function. People with diastolic dysfunction have significantly reduced e' velocity as their left ventricles are stiff and non-compliant. The ratio between $\mathrm{E}$ wave and e' wave (E/e') has been well validated to predict the left atrial filling pressure, especially in patients with $\mathrm{HFpEF}$, even in the presence of atrial fibrillation. ${ }^{15,16}$ The tricuspid regurgitation velocity measured with continuous-wave Doppler ultrasonography can be used to estimate the pulmonary artery systolic pressure, which may be elevated in patients with HFpEF. ${ }^{17}$ The pattern of left atrial filling from pulmonary veins can also be used to determine diastolic function; however, it is challenging to obtain these signals using transthoracic echocardiography as pulmonary veins are the furthest structures from the ultrasonography probe.

\section{Other investigations}

If the initial investigation with comprehensive echocardiography is not diagnostic; despite a high index of suspicion for HFpEF, other investigations such as stress echocardiography (exercise or pharmacological), cardiac magnetic resonance imaging (MRI) and right heart catheterisation may be considered. Stress echocardiography may unmask the diastolic dysfunction and high left heart filling pressure that are not apparent at rest. ${ }^{18,19}$ Cardiac MRI may also be useful to assess left ventricular relaxation, myocardial mechanics, flow dynamics and the extent of myocardial fibrosis; however, it is not widely available, and some of the methods are still experimental. Sleep studies and pulmonary function tests should also be considered in selected patients with risk factors suggestive of sleep apnoea and pulmonary diseases.

\section{Case studies}

\section{CASE 1}

Mr ET is a retiree aged 70 years who has been in and out of hospital as a result of frequent exacerbation of heart failure over the past few years. He is currently functioning at New York Heart Association Class IV. He has a history of longstanding hypertension, obesity, obstructive sleep apnoea, mixed obstructive and restrictive lung disease, and permanent atrial fibrillation.

On review of his echocardiograms throughout the years, it was noted that even before he developed the clinical syndrome of heart failure, there were echocardiographic signs of impaired relaxation (mild diastolic dysfunction) as well as an enlarged left atrium on the echocardiograms performed since 2010 (Figure 6A). His left ventricular systolic function has always been preserved. He was also in sinus rhythm in 2010. Seven years later, he developed overt heart failure as well as two well-known consequences of HFpEF: atrial fibrillation and pulmonary hypertension. His latest echocardiogram showed preserved left ventricular systolic function with signs of severe diastolic dysfunction (Figure 6B). This case highlights the importance of early recognition of diastolic dysfunction and prevention of future heart failure by aggressive risk factor control.

\section{CASE 2}

$\mathrm{Mr} \mathrm{RO}$ is a man aged 68 years who presented with acute heart failure and pulmonary oedema. He had atrial flutter on presentation, which was cardioverted to sinus rhythm electrically. His ECG had poor anterior R wave progression (Figure 5A) while his echocardiogram showed moderate to severe LVH (Figure 5B) with preserved left ventricular systolic function. The Doppler haemodynamic assessment in sinus rhythm revealed a restrictive filling pattern or severe diastolic dysfunction (Figure 5C). He had an increased level of serum light chain proteins and plasma cell dyscrasia. The repeat echocardiography with global longitudinal strain showed typical apical sparing pattern consistent with cardiac amyloidosis (Figure 5D). The diagnosis was confirmed with colonic mucosal biopsies, which showed amyloid infiltration, and he was scheduled to have chemotherapy.

\section{Management}

There is currently no single effective, evidence-based treatment for established HFpEF. Multiple clinical trials using different classes of heart failure therapy (eg angiotensin-converting enzyme inhibitors, angiotensin receptor blockers, beta-blockers and aldosterone antagonists), which are effective in HFrEF, have failed to show significant benefits in patients with HFpEF. ${ }^{20-23}$ Possible reasons for this failure is the heterogeneity of the HFpEF itself, the loose definition of $\mathrm{HFpEF}$ and inappropriate recruitment of patients in the trials. ${ }^{24}$ For example, diastolic function was normal in one-third of gradable patients who were recruited in a landmark HFpEF trial, Treatment of Preserved Cardiac Function Heart Failure with an Aldosterone Antagonist (TOPCAT), inevitably diluting the final result. ${ }^{22,25}$

In the absence of evidence-based therapy, the management of HFpEF should be directed towards treatment of underlying pathologies and management of symptoms. Diuretics can be used to reduce congestion and improve symptoms. Low-dose spironolactone is recommended to reduce hospital admission on the basis of the TOPCAT trial results. ${ }^{22}$

Controlling and managing comorbidities (ie hypertension, obesity, obstructive sleep apnoea and diabetes) as well as preventing conditions that worsen diastolic function (ie atrial fibrillation and tachycardia) are the cornerstones of HFpEF management strategies. Tachycardia disproportionately shortens the diastolic time, while atrial fibrillation 

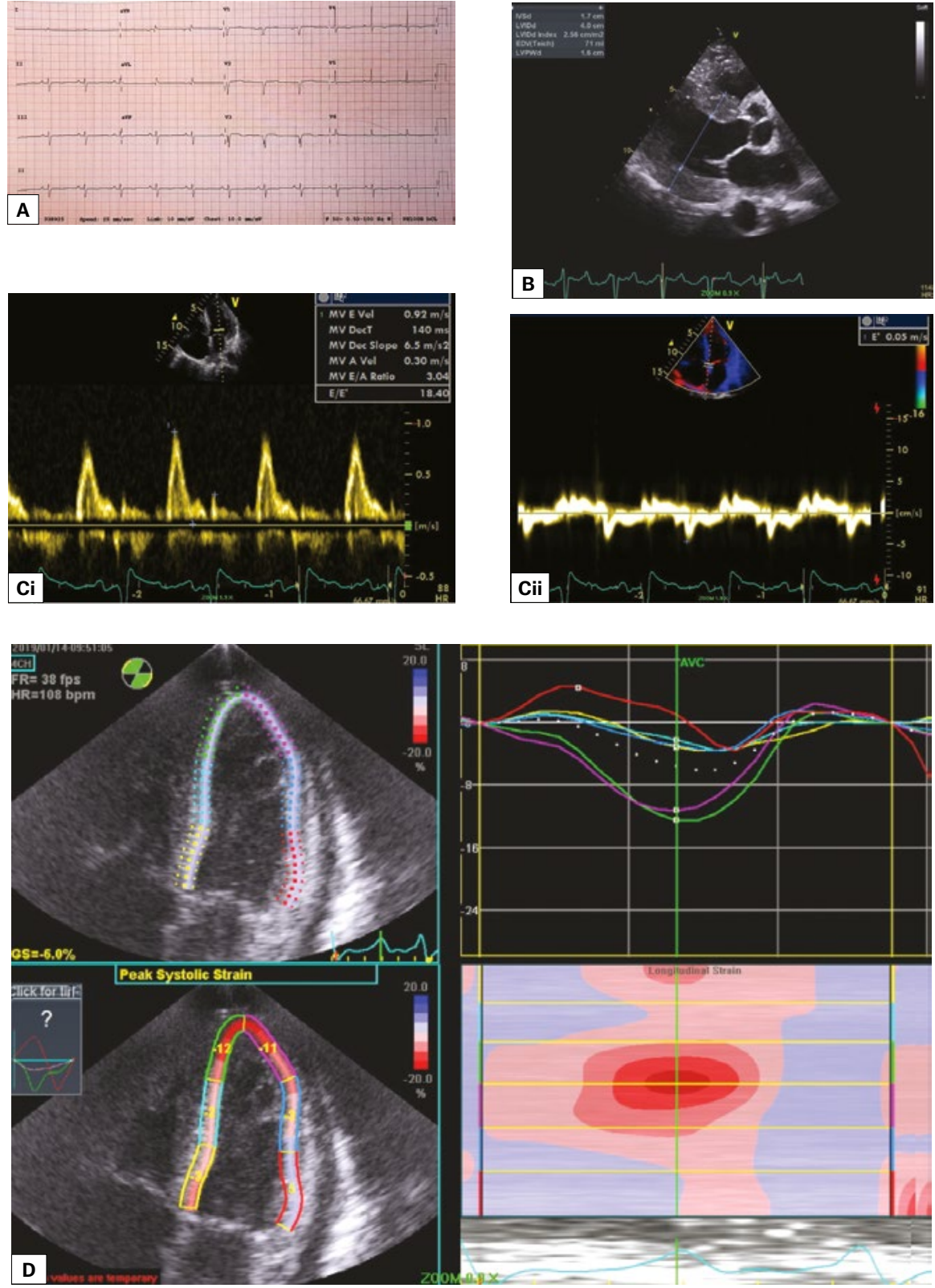

Figure 5 A. Electrocardiogram showing relatively poor R progression without the typical LVH strain pattern; B. Parasternal long-axis view on echocardiogram showing concentric ventricular hypertrophy (LVH) with septal thickness of $1.7 \mathrm{~cm}$ and posterior wall thickness of $1.6 \mathrm{~cm} ; \mathbf{C i}$, ii. Restrictive filling pattern with $E / A>3$ and high left atrial filling pressure with $E / e^{\prime}=18.4 ;$ D. Global longitudinal strain showing apical sparing, which is highly suggestive of amyloid heart disease.

causes loss of atrial contraction; both can be detrimental in patients with diastolic dysfunction. Valvular heart disease such as aortic stenosis should be monitored closely, and early intervention should be considered once evidence of pressure strain (eg LVH) is detected.

A sedentary lifestyle and lack of physical exercise are directly proportional to the rate of hospitalisation and mortality in patients with $\mathrm{HFpEF}{ }^{26,27}$ Therefore, patients should be educated about the benefits of increasing physical activity and referred to cardiac rehabilitation programs. Heart failure education and support through peer groups and specialist heart failure nurses also have been shown to improve quality of life and reduce hospital admissions. ${ }^{28}$ The traditional model of a cardiologistled, hospital-based heart failure clinic may not be the answer to this epidemic, while a multidisciplinary team-approach of management coordinated by general practitioners may benefit many patients with this condition. ${ }^{29}$ Timely referral to cardiologists to exclude cardiac ischaemia and significant valvular heart disease is also very important. Early recognition of diastolic dysfunction and aggressive management of risk factors may prevent many patients from developing overt heart failure. ${ }^{27}$ Even for patients with overt HFpEF, management of comorbidities is as important as management of heart failure itself, and generalists have important roles in diagnosis and management of HFpEF.

\section{Conclusion}

HFpEF due to left ventricular diastolic dysfunction is very common in both community and hospital settings. It is associated with morbidity and mortality approximately equal to that of HFrEF but is much harder to diagnose because of the complexity of interpretation of diastolic function on echocardiography. A high index of clinical suspicion is required. Delay in diagnosis leads to advanced disease with a poor prognosis, while early detection may allow treatment of underlying causes. Clinical clues as well as echocardiography should be used for early diagnosis of this condition. Increased awareness of this condition among the public, as well as within primary healthcare, is essential to halt the global epidemic of HFpEF. Identifying individuals who are at risk of developing this condition and effecting prevention using education, physical exercise and aggressive risk factor control are the keys to achieving this goal. 


\section{Key points in HFpEF management}

Effective management of HPpEF relies on:

- early diagnosis

- awareness of high-risk patients (ie elderly female patients presenting with wheeze and cough are more likely to have HFpEF than asthma)

- assessment for symptoms of heart failure in patients with risk factors (hypertension, diabetes, obesity, chronic kidney disease, obstructive sleep apnoea)

- early referral for echocardiography, including assessment of diastology

- testing for BNP level in suspected patients

- symptom management

- diuretics (including spironolactone)

- physical exercise

- salt and water restriction

- support and education through peer groups and specialist heart failure nurses

- prevention of deterioration

- prevention of arrhythmia and tachycardia

- exclusion/treatment of sleep apnoea

- aggressive blood pressure and diabetes control if present

- weight loss
- avoidance of management pitfalls

- assessment for underlying coronary artery disease and valvular disease, which may be missed

- assessment for restrictive cardiomyopathies (eg cardiac amyloidosis) that may be missed in early stages

- referral to specialist cardiology services if in doubt.

\section{Authors}

Pyi Naing MBBS, MPhil, GDipEcho, Senior Registrar, Royal Darwin Hospital, NT; MPhil Candidate, University of Notre Dame Australia (Fremantle), WA; Associate Lecturer, Flinders University, SA. kopyinaing@gmail.com

Douglas Forrester BSc, BMedSci, BMBS, PhD, MRCP (UK), Consultant Respiratory Physician, Royal Darwin Hospital, NT

Nadarajah Kangaharan MBBS, FRACP, Consultant Cardiologist, Co-Director of Division of Medicine, Royal Darwin Hospital, NT

Aruna Muthumala, MBBS, MD, FRACP, Consultant Physician, Royal Darwin Hospital, NT

Su Mon Myint MBBS, General Practice Trainee Registrar, Danila Dilba Health Service, NT

David Playford MBBS, PhD, FRACP, Professor of Cardiology, University of Notre Dame Australia (Fremantle), WA

Competing interests: None.

Funding: None.

Provenance and peer review: Not commissioned, externally peer reviewed.

\section{References}

1. Atherton JJ, Sindone A, De Pasquale CG, et al. National heart foundation of Australia and cardiac society of Australia and New Zealand: Guidelines for the prevention, detection, and management of heart failure in Australia 2018. Heart Lung Circ 2018;27(10):1123-208. doi: 10.1016/j. hlc.2018.06.1042.

2. Nagueh $\mathrm{SF}$, Smiseth $\mathrm{OA}$, Appleton $\mathrm{CP}$, et al. Recommendations for the evaluation of left ventricular diastolic function by echocardiography: An update from the American society of echocardiography and the European association of cardiovascular imaging. J Am Soc Echocardiogr 2016;29(4):277-314. doi: 10.1016/j.echo.2016.01.011.

3. Redfield MM, Jacobsen SJ, Burnett JC Jr, Mahoney DW, Bailey KR, Rodeheffer RJ. Burden of systolic and diastolic ventricular dysfunction in the community: Appreciating the scope of the heart failure epidemic. JAMA 2003;289(2):194-202. doi: 10.1016/S1062-1458(03)00178-8.

4. Guazzi M, Gomberg-Maitland M, Arena R Pulmonary hypertension in heart failure with preserved ejection fraction. J Heart Lung Transplant 2015;34(3):273-81. doi: 0.1016/j. healun.2014.11.003.

5. Strange G, Playford D, Stewart S, et al. Pulmonary hypertension: Prevalence and mortality in the Armadale echocardiography cohort. Heart 2012;98(24):1805-11. doi: 10.1136/ heartjnl-2012-301992.

6. Owan TE, Hodge DO, Herges RM, Jacobsen SJ, Roger VL, Redfield MM. Trends in prevalence and outcome of heart failure with preserved ejection fraction. N Engl J Med 2006;355(3):251-59. doi: 10.1056/NEJMoa052256.

7. Taylor CJ, Valenti L, Britt $\mathrm{H}$, et al. Management of chronic heart failure in general practice in Australia. Aust Fam Physician 2016;45(11):823-27.
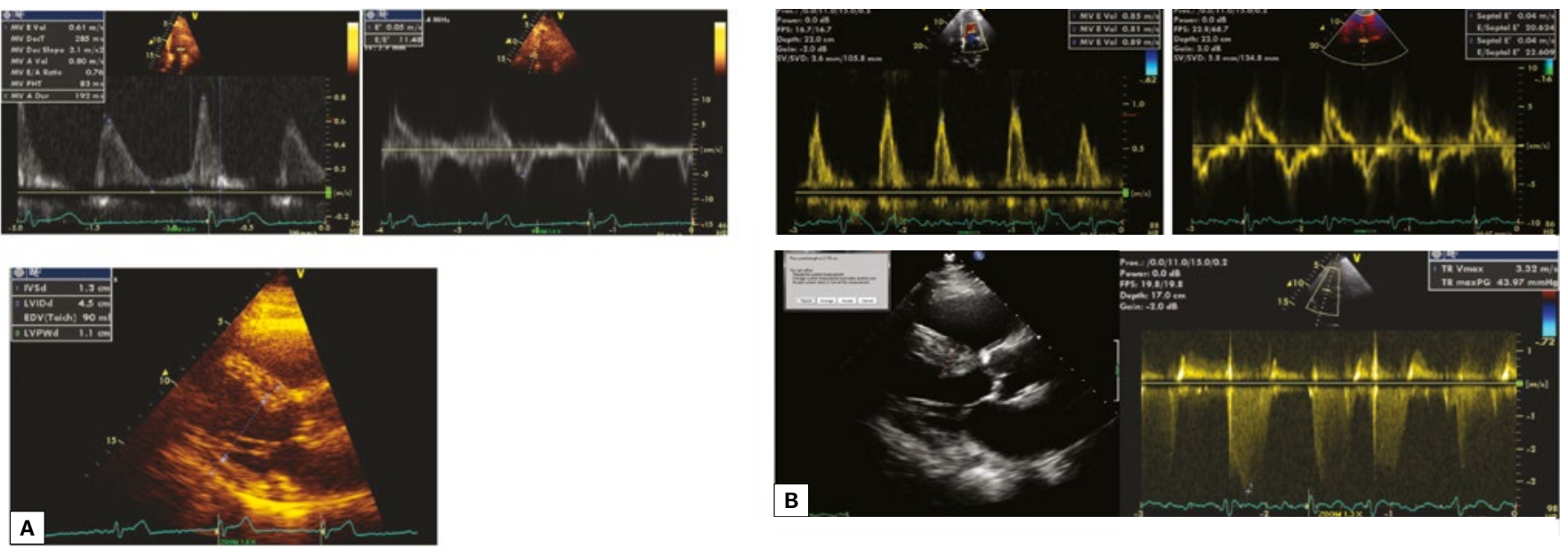

Figure 6 A. The patient's echocardiogram in 2010 showed impaired relaxation pattern (reverse E/A) and borderline elevated E/e' (upper images), and mild left ventricular hypertrophy with septal wall measuring $1.3 \mathrm{~cm}$ (lower image); B. In 2017, the patient had a restrictive left ventricular filling pattern as well as high left ventricular filling pressure $\left(E / e^{\prime}=22\right)$ with atrial fibrillation and pulmonary hypertension as consequences. The lower left image shows left ventricular hypertrophy with septal wall thickness of $2 \mathrm{~cm}$. The lower right image shows elevation of tricuspid regurgitation velocity, which can be used to estimate pulmonary artery systolic pressure. 
8. Taylor CJ, Harrison C, Britt H, Miller G, Hobbs FDR. Heart failure and multimorbidity in Australian general practice. J Comorb 2017;7(1):44-9. doi: 10.15256/joc.2017.7.106.

9. Deaton C, Benson J. Time for correct diagnosis and categorisation of heart failure in primary care. Br J Gen Pract 2016;66(652):554-55. doi: 10.3399/bjgp16X687649

10. Bishu K, Redfield MM. Acute heart failure with preserved ejection fraction: Unique patient characteristics and targets for therapy. Curr Heart Fail Rep 2013;10(3):190-97. doi: 10.1007/s11897013-0149-5.

11. Tsutsui H, Tsuchihashi-Makaya M, Kinugawa S. Clinical characteristics and outcomes of heart failure with preserved ejection fraction: Lessons from epidemiological studies. J Cardiol 2010;55(1):13-22. doi: 10.1016/j.jjcc.2009.09.003.

12. Harada E, Mizuno Y, Kugimiya F, et al. B-type natriuretic peptide in heart failure with preserved ejection fraction- relevance to age-related left ventricular modeling in Japanese. Circ J 2017;81(7):1006-13. doi: 10.1253/circj.CJ-16-1282.

13. Meijers WC, van der Velde AR, de Boer RA. Biomarkers in heart failure with preserved ejection fraction. Neth Heart J 2016;24(4):252-58. doi: 10.1007/s12471-016-0817-7.

14. Dokainish $\mathrm{H}$. Left ventricular diastolic function and dysfunction: Central role of echocardiography. Glob Cardiol Sci Pract 2015;2015:3. doi: 10.5339/ gcsp.2015.3.

15. Matsushita K, Minamishima T, Goda A, et al. Comparison of the reliability of $E / E$ ' to estimate pulmonary capillary wedge pressure in heart failure patients with preserved ejection fraction versus those with reduced ejection fraction. Int J Cardiovasc Imaging 2015;31(8):1497-502. doi: 10.1007/s10554-015-0718-7.

16. Senechal M, O'Connor K, Deblois J, et al. A simple doppler echocardiography method to evaluate pulmonary capillary wedge pressure in patients with atrial fibrillation. Echocardiography 2008;25(1):57-63. doi: 10.1111/j.15408175.2007.00555.x.

17. Yock PG, Popp RL. Noninvasive estimation of right ventricular systolic pressure by doppler ultrasound in patients with tricuspid regurgitation. Circulation 1984;70(4):657-62.

18. Obokata M, Kane GC, Reddy YN, Olson TP, Melenovsky V, Borlaug BA. Role of diastolic stress testing in the evaluation for heart failure with preserved ejection fraction. Circulation 2017;135(9):825-38. doi: 10.1016/S07351097(17)34268-7.
19. Takagi T. Diastolic stress echocardiography. J Echocardiogr 2017;15(3):99-109. doi: 10.1007/ s12574-017-0335-7.

20. Cleland JG, Tendera M, Adamus J, Freemantle N, Polonski L, Taylor J; PEP-CHF Investigators. The perindopril in elderly people with chronic heart failure (PEP-CHF) study. Eur Heart J 2006;27(19):2338-45. doi: 10.1093/eurheartj/ehl250

21. Yusuf S, Pfeffer MA, Swedberg K, et al. Effects of candesartan in patients with chronic heart failure and preserved left-ventricular ejection fraction: The CHARM-Preserved Trial. Lancet 2003;362(9386):777-81. doi: 10.1016/S01406736(03)14285-7

22. Pitt B, Pfeffer MA, Assmann SF, et al. Spironolactone for heart failure with preserved ejection fraction. N Engl J Med 2014;370(15):1383-92. doi: 10.1056/ NEJMoa1313731.

23. Hernandez AF, Hammill BG, O'Connor CM, Schulman KA, Curtis LH, Fonarow GC. Clinical effectiveness of beta-blockers in heart failure: Findings from the OPTIMIZE-HF (Organized Program to Initiate Lifesaving Treatment in Hospitalized Patients with Heart Failure) Registry. J Am Coll Cardiol 2009;53(2):184-92. doi: 10.1016/j.jacc.2008.09.031

24. Roh J, Houstis N, Rosenzweig A. Why don't we have proven treatments for HFpEF? Circ Res 2017;120(8):1243-45. doi: 10.1161/ CIRCRESAHA.116.310119.

25. Shah AM, Shah SJ, Anand IS, Sweitzer NK, O'Meara E, Heitner JF, et al. Cardiac structure and function in heart failure with preserved ejection fraction: Baseline findings from the echocardiographic study of the Treatment of Preserved Cardiac Function Heart Failure with an Aldosterone Antagonist trial. Circ Heart Fail 2014;7(1):104-15.

26. Hegde SM, Claggett B, Shah AM, et al. Physical activity and prognosis in the TOPCAT trial (Treatment of Preserved Cardiac Function Heart Failure With an Aldosterone Antagonist). Circulation 2017;136(11):982-92. doi: 10.1161/ CIRCULATIONAHA.117.028002.

27. Shah SJ. Sedentary lifestyle and the risk for HFpEF: Are 'huff-puff health clubs' the answer? J Am Coll Cardiol 2017;69(4):1143-46. doi: 10.1016/j.jacc.2017.01.010.

28. Betihavas V, Newton PJ, Du HY, et al. Australia's health care reform agenda: Implications for the nurses' role in chronic heart failure management. Aust Crit Care 2011;24(3):189-97. doi: 10.1016/j. aucc.2010.08.003.
29. Scott I, Jackson C. Chronic heart failure management in Australia: Time for general practice centred models of care? Aust Fam Physician 2013;42(5):343-46. 